\title{
Denseness and Zariski denseness of Jones braid representations
}

\author{
GREG KUPERBERG
}

\begin{abstract}
Using various tools from representation theory and group theory, but without using hard classification theorems such as the classification of finite simple groups, we show that the Jones representations of braid groups are dense in the (complex) Zariski topology when the parameter $t$ is not a root of unity. As first established by Freedman, Larsen and Wang, we obtain the same result when $t$ is a nonlattice root of unity, other than one initial case when $t$ has order 10. We also compute the real Zariski closure of these representations (meaning, the closure in Zariski closure of the real Weil restriction). When such a representation is indiscrete in the analytic topology, then its analytic closure is the same as its real Zariski closure.
\end{abstract}

\section{Introduction}

In this article we will study representations of braid groups associated with the Jones polynomial $J(L, t)$. Our question is to determine the closures of these representations, which are then Lie groups. Freedman, Larsen and Wang [4] computed these closures in the important case where $t=\exp (2 \pi i / r)$ is a principal root of unity. In this case, the (reduced) braid representations are unitary, and the braids can be interpreted as quantum circuits. Freedman, Larsen and Wang established that the Jones representations are eventually dense if $r=5$ or $r \geq 7$. This has the important corollary that these representations are universal for quantum computation.

In this article $t$ will usually be a complex number which is not a root of unity. Although the Jones polynomial is not directly a model of quantum computation for these values of $t$, the closure of the braid group representation is still interesting for related questions in complexity theory; see Ahargonov, Arad, Eban and Landau [1]. Also, we will say more about the Zariski closure of the braid group action in the target group $\operatorname{GL}(N, \mathbb{C})$, rather than the closure in the usual topology. Switching to the Zariski topology simplifies the question, and yet in many cases it does not change the question very much. To be precise, we will consider two Zariski topologies on $\operatorname{GL}(N, \mathbb{C})$ and $\operatorname{SL}(N, \mathbb{C})$. The usual Zariski topology is generated by polynomials in the complex matrix entries, and 
we will call it the complex Zariski topology. We will also consider the real Zariski topology generated by polynomials in the real and imaginary parts of the complex matrix entries. (In the more current language of algebraic geometry, the real Zariski topology is the Zariski topology on the real points of the Weil restriction $\operatorname{Res}_{\mathbb{C} / \mathbb{R}}(\operatorname{GL}(N))$.)

Our main results are as follows:

Theorem 1.1 Let $t \in \mathbb{C}$ be a nonzero complex number, and let $n \geq 4$ and $c \geq 0$ be integers. Let $X(n \cdot 1, c, t)$ be the (reduced) Jones representation of the braid group $B_{n}$ with quantum parameter $t, n$ ordinary strands, and the strand color $c$ at infinity. If $t$ is not a root of unity, or if $t$ is a root of unity of order $r \geq 5$ and $r \neq 6,10$, then the representation is complex Zariski dense in $\operatorname{SL}(X(n \cdot 1, c, t))$. When $r=10$, the same is true with $n+c \geq 5$.

A complex number $t$ is a lattice root of unity if it is a root of unity of order 1, 2, 3, 4 or 6 . (So that the ring $\mathbb{Z}[t]$ is a discrete lattice in $\mathbb{C}$.) Theorem 1.1 is trivially true when $t=2$ or $t=3$, because then the reduced Jones representations are all 1-dimensional. It is false when $t=1$ because the Jones representation is large but trivial; and it is false when $t=4$ or $t=6$ for less trivial reasons. When $r=10$, the projective image of the braid group action on $X(3 \cdot 1,1, t)=X(4 \cdot 1,0, t)$ lies in $\mathrm{PSU}(2) \cong \mathrm{SO}(3)$ and is that of the icosahedral group.

Corollary 1.2 Assuming the hypotheses of Theorem 1.1:

(1) If $t \in \mathbb{C}$ and $t \notin \mathbb{R} \cup S^{1}$ is complex, but neither real nor norm 1, then the action of $B_{n}$ is real Zariski dense in $\operatorname{SL}(X(n \cdot 1, c, t))$.

(2) If $t \in \mathbb{R} \backslash\{0, \pm 1\}$, then the action of $B_{n}$ is real Zariski dense in $\operatorname{SL}(X(n$. $\left.1, c, t)_{\mathbb{R}}\right)$.

(3) If $|t|=1$ but $t$ is not a root of unity, then $B_{n}$ acts densely in $\operatorname{SU}(X(n \cdot 1, c, t))$ in the analytic topology. Here $\operatorname{SU}(X(n \cdot 1, c, t))$ is defined using the invariant, but typically indefinite, Hermitian structure on $X(n \cdot 1, c, t)$.

(4) If $t$ is a nonprincipal nonlattice root of unity, then the action of $B_{n}$ is real Zariski dense in $\mathrm{SU}(X(n \cdot 1, c, t))$.

(5) If $t$ is a principal nonlattice root of unity, then the action of $B_{n}$ is analytically dense in the compact group $\mathrm{SU}(X(n \cdot 1, c, t))$.

(6) If the action of $B_{n}$ on $X(n \cdot 1, c, t)$ is analytically indiscrete, then in cases 1,2 and 4 above, the analytic closure is the same as the real Zariski closure. 
Theorem 1.3 Let $c_{1}, c_{2}, \ldots, c_{\ell}$ be distinct nonnegative integers and let $t$ and $n$ be as in Theorem 1.1. Suppose that for each $k$, the action of $B_{n}$ is dense in one of the groups $G_{k}$ listed in Theorem 1.1 or Corollary 1.2 in either the complex or real Zariski topology. Its diagonal action is dense in the same topology in

$$
G_{1} \times G_{2} \times \cdots \times G_{\ell} .
$$

Case 5 of Corollary 1.2 (plus the analogous case of Theorem 1.3) is exactly the theorem of Freedman, Larsen and Wang. Our argument is not particularly simpler than theirs, but one simplification is that our proof does not use the classification of finite simple groups. Also, some of our ideas and related results were also found by Aharonov, Arad, Eban and Landau [1]. However, some of our techniques, such as the emphasis on the Zariski topology, is new.

Besides the specific results, our purpose is to describe a set of tools to establish the closure of representations of groups such as braid groups. Our tools are described without proof in Section 3 and established in Section 4. We think that they could be used to further investigate the images of braid group actions, such as those coming from the colored Jones polynomial or other quantum link invariants. (The Jones polynomial is associated to defining representation of the Lie algebra $\mathrm{sl}(2)$. There is a polynomial invariant for every simple Lie algebra, colored by its irreducible representations.)

As an example use of our tools, case 4 of Corollary 1.2 follows from case 5 , the FLW theorem, using the fact that the complex Zariski topology is preserved by Galois automorphisms of $\mathbb{C}$. The action in case 4 is then dense if and only if there is a braid whose action is an elliptic element of infinite order.

In Section 7, we will discuss examples where the braid group acts discretely or indiscretely on $X(3 \cdot 1,1, t)=X(4 \cdot 1,0, t)$ and $X(4 \cdot 1,2, t)$ in the analytic topology. Again, case 6 of Corollary 1.2 says that if $B_{n}$ acts indiscretely, then it is dense in its real Zariski closure.

Theorem 1.4 The following is a complete classification of when $B_{3}$ acts discretely on $X(3 \cdot 1,1, t)$, or equivalently when $B_{4}$ acts discretely on $X(4 \cdot 1,0, t)$, in the cases $t \in \mathbb{R}$ and $|t|=1$.

(1) Let $t \neq 0,-1$ be real. Then $B_{3}$ acts discretely in $\operatorname{PGL}\left(X(3 \cdot 1,1, t)_{\mathbb{R}}\right)$ in the following cases:

$$
t<0 \quad t+t^{-1} \geq 3 \quad t+t^{-1}=1+2\left(\cos \frac{2 \pi}{n}\right) .
$$


(2) Let $t=\exp (i \theta)$ with $0<|\theta|<2 \pi / 3$. Then $B_{3}$ acts discretely in the compact group $\operatorname{PSU}(X(3 \cdot 1,1, t))$ if and only if $|\theta|=\pi-(2 \pi / n)$.

(3) Let $t=\exp (i \theta)$ with $\pi>|\theta|>2 \pi / 3$. Then $B_{3}$ acts discretely in the noncompact group $\operatorname{PSU}(X(3 \cdot 1,1, t))$ if and only if $|\theta|=\pi-(2 \pi / n)$.

(4) If $t$ satisfies

$$
t+t^{-1}=1+2 \cos \frac{2 \pi}{7}
$$

then $B_{4}$ acts indiscretely in $\operatorname{PGL}\left(X(4,2, t)_{\mathbb{R}}\right)$.

Finally in Section 7, we will discuss the following corollary of Theorem 1.1. It is used by the author in a previous paper [8] to establish that certain values of the Tutte polynomial are \#P-hard to estimate multiplicatively.

Corollary 1.5 The edge operators $A_{j, y}$ and $B_{j, x}$ defined in [8], using all $x, y \neq 1$, generate $\operatorname{PSL}\left(V(n)_{\mathbb{R}}\right)$, where $V(n)_{\mathbb{R}}$ is the real skein space of the Tutte polynomial on $n$ vertices with Potts model parameter $q \geq 4$.

Acknowledgements The author would like to thank Scott Carnahan, Nathan Dunfield, Mike Freedman, Jim Humphreys, Misha Kapovich, Michael Larsen and David Speyer for useful discussions.

This material is based upon work supported by the National Science Foundation under grant number 0606795

\section{Definitions for the main results}

\subsection{Geometry}

We the definitions needed in the statements of the main results: If $\mathbb{F}$ is an infinite field, the Zariski topology on the vector space $\mathbb{F}^{N}$, or any subset of $\mathbb{F}^{N}$, is the coarsest topology in which the solution set of a polynomial equation is closed. If we interpret $\operatorname{SL}(N, \mathbb{C})$ as a subset of $\mathbb{C}^{N^{2}}$, it inherits a complex Zariski topology. If instead we interpret it as a subset of $\mathbb{R}^{2 N^{2}}$, it inherits a real Zariski topology. If we further restrict to $\operatorname{SU}(P, Q) \subset \operatorname{SL}(N, \mathbb{C})$ with $P+Q=N$, then the real and complex Zariski topologies agree. Likewise $\operatorname{SL}(N, \mathbb{R})$ has the same Zariski topology, whether it is viewed as a subset of $\mathbb{R}^{N^{2}}$ or as a subset of $\operatorname{SL}(N, \mathbb{C})$ with either of its Zariski topologies.

The analytic topology on any subset of $\mathbb{R}^{N}$ or $\mathbb{C}^{N}$ is the usual topology used in calculus and in most mathematics. 
If $G$ is a topological group with a closed subgroup $H$ and another subgroup $\Gamma$, then we say that $\Gamma$ is dense in $H$ if $\Gamma \cap H$ is dense in $H$.

An operator $x$ on a vector space $X$ is elliptic if it is diagonalizable, and if its eigenvalues are all on the unit circle.

\subsection{Quantum algebra}

The Jones polynomial can be defined from the Kauffman bracket, which is defined by these skein relations:

$$
\left.Y=-t^{1 / 4}\right)\left(-t^{-1 / 4} \frown \quad \bigcirc=-t^{1 / 2}-t^{-1 / 2} .\right.
$$

For the moment, we let $t^{1 / 4} \in \mathbb{F} \backslash\{0\}$ for a field $\mathbb{F}$ of characteristic 0 . Note also that the Kauffman bracket is only invariant under two of the three Reidemeister moves; it gains a factor of $t^{3 / 4}$ under the first Reidemeister move.

Given nonnegative integers $n$ and $c$ of the same parity, define the skein space $W(n \cdot 1, c, t)$ to be the vector space of formal linear combinations of planar matchings in a rectangle, with $n$ points on the left and $c$ points on the right. The elements of $W(n \cdot 1, c, t)$ are skeins. The $c$ points on the right are together called a clasp. We set to 0 those matchings that have a U-turn at the right side:
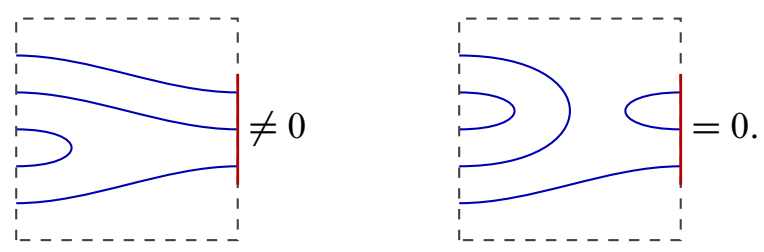

The braid group $B_{n}$ acts on $W(n \cdot 1, c, t)$ by braiding the clasps and then expanding crossings:
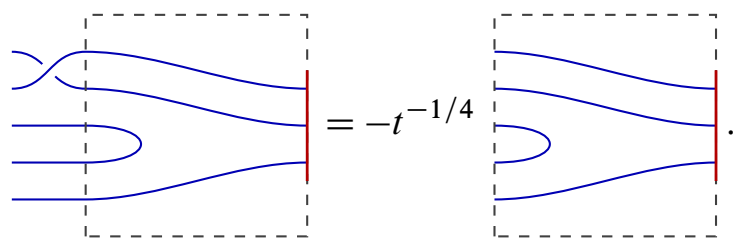

When $t$ is not a root of unity, we let $X(n \cdot 1, c, t)=W(n \cdot 1, c, t)$; this skein space with the action of $B_{n}$ is then projectively equivalent to the Jones representation. Projective equivalence does not change the density properties of interest to us; see Lemma 3.7. 
We would like to define $W(n \cdot 1, c, t)_{\mathbb{F}}$ over a field $\mathbb{F}$ that does not contain $t^{1 / 4}$ or even $t^{1 / 2}$. To this end, we first let each braid generator be a right-handed crossing times $t^{1 / 4}$ :

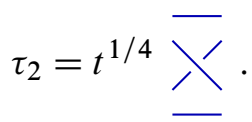

Second, we bicolor the complementary regions of a matching in the rectangle, so that regions are alternately black and white and the top region is white:

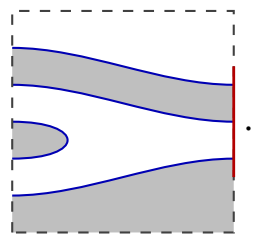

Then we can define a matching to be even or odd according to the number of black regions in the coloring. If we multiply the odd matchings by $t^{1 / 2}$, the result is a basis for which action of $B_{n}$ is defined over $\mathbb{F}$.

The skein space $W(n \cdot 1, c, t)$ carries a natural bilinear form which is used to define $X(n \cdot 1, c, t)$ when $t$ is a root of unity. If $r$ is the order of $t$, we assume that $c \leq r-2$. Given two skeins $A$ and $B$, we pair them by sewing their rectangles together at both ends. Where the clasps meet, we insert a special skein called a Jones-Wenzl projector (defined in Section 5):

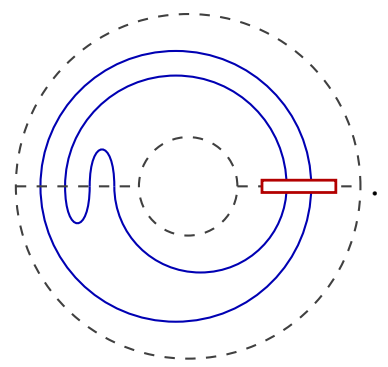

The Kauffman bracket value of this diagram is then the value of the pairing. When $t$ is a root of unity, the form $\langle A, B\rangle$ has a kernel, and $X(n \cdot 1, c, t)$ is defined by annihilating this kernel so that $\langle A, B\rangle$ is nondegenerate in the quotient.

If a skein $A$ has crossings, then we define its reflection $\bar{A}$ by switching left and right crossings. The form $\langle\bar{A}, B\rangle$ is invariant under the action of $B_{n}$, and when $|t|=1$ it is Hermitian. 


\section{The tools}

Lemma 3.1 (Adjoint) Let $\Gamma$ be a subgroup of a connected, simple Lie group $G$. Then $\Gamma$ is dense if and only if the Lie algebra $\mathfrak{g}$ is irreducible under the adjoint action of $\Gamma$ and $\Gamma$ is indiscrete.

Lemma 3.1 reduces the question of computing the closure of a group in a Lie group to representation theory. The lemma holds in both the analytic topology on $G$ and in the Zariski topology, defined below. Henceforth we will assume that all representations and other vector spaces that we use are finite-dimensional.

In light of Lemma 3.1, we define a subgroup $\Gamma \subset G$ of a simple Lie group $G$ to be adjoint-irreducible if $\mathfrak{g}$ is $\Gamma$-irreducible, but $\Gamma$ is not necessarily dense. Thus, Lemma 3.1 by itself establishes case 6 of Corollary 1.2: If $\Gamma$ (or an action of $\Gamma$ ) is real Zariski dense, then it is adjoint-irreducible. If in addition it is indiscrete in the analytic topology, it is then dense in that topology.

Lemma 3.2 (Zariski) If $\Gamma$ is a Zariski-dense subgroup of a group $G$, then every admissible representation $V$ of $G$ decomposes in the same way as a $G$-representation and as a $\Gamma$-representation.

Lemma 3.2 uses the Zariski topology as a convenient converse, using closures of groups to help compute decompositions of representations. In order to define the Zariski topology on a group $G$, we first choose a class $\mathcal{V}$ of admissible representations over an infinite field $\mathbb{F}$. The $\mathcal{V}$ should be closed under passing to duals, direct sums, tensor products, and subrepresentations (because taking this closure does not change the resulting topology). Given $\mathcal{V}$, the Zariski topology on $G$ is the topology whose closed sets are generated by solutions to polynomial equations in the entries of the matrices of representations $V \in \mathcal{V}$, using some basis for $V$.

If $G \subseteq \mathrm{GL}(N, \mathbb{F})$, then one standard admissible class is the set of representations generated from the defining representation $V=\mathbb{F}^{N}$. In particular, this $V$ generates the adjoint representation. We are also interested in the real Zariski topology on $\operatorname{GL}(N, \mathbb{C})$. This topology is generated by the same representation $V=\mathbb{C}^{N}$, but interpreted as a real vector space $V \cong \mathbb{R}^{2 N}$ instead.

Lemma 3.3 (Compact) A subgroup $G \subseteq \mathrm{U}(N)$ is analytically closed if and only if it is Zariski closed.

Lemma 3.3 establishes case 5 of Corollary 1.2 from Theorem 1.1. 
Lemma 3.4 (Closure) If $f: G \rightarrow H$ is an algebraic homomorphism between algebraic groups, then the image $f(G)$ is Zariski closed.

An algebraic group is an affine algebraic variety that is also a group with an algebraic group law. Not every group with a Zariski topology in our sense is an algebraic group. However, any Zariski-closed subgroup of $\operatorname{GL}(N, \mathbb{F})$ is an algebraic group, and this covers all cases of interest to us.

Lemma 3.4 is a major reason to trust the Zariski topology in our context. Indeed, the Zariski topology is more trustworthy than the analytic topology, in the sense that Lemma 3.4 is not always true in the analytic topology for noncompact groups.

Example Consider the ring $\mathbb{Z}[\sqrt{3}]$ (or the ring of integers of any other real Galois number field larger than $\mathbb{Q})$. Let $\Gamma$ be the group of pairs of matrices $(x, \bar{x})$ in

$$
G=\operatorname{SL}(2, \mathbb{R}) \times \operatorname{SL}(2, \mathbb{R}),
$$

where $x \in \operatorname{SL}(2, \mathbb{Z}[\sqrt{3}])$ and $\bar{x}$ is its Galois conjugate. Then $\Gamma$ is a discrete subgroup of $G$ because it is Zariski dense but not analytically dense. It is not analytically dense because the product of two entries of $x$ and $\bar{x}$ in the same position is an ordinary integer. On the other hand the projection of $\Gamma$ onto either factor is $\operatorname{SL}(2, \mathbb{Z}[\sqrt{3}])$, which is dense. This is a counterexample not only to Lemma 3.4, but to its use in combination with Lemma 3.5 below.

Lemma 3.5 (Diagonal; P Hall [6]) Suppose that each of $G_{1}, G_{2}, \ldots, G_{\ell}$ is an adjoint simple Lie group or a nonabelian finite simple group, and suppose that

$$
H \subseteq G=G_{1} \times G_{2} \times \cdots \times G_{\ell}
$$

is a closed subgroup that surjects onto each factor $G_{k}$. Then $H$ is a diagonal subgroup of $G$.

A Lie group $G$ is adjoint simple if its Lie algebra $\mathfrak{g}$ is simple, and if it is connected and has no center. (See the remarks after Lemma 3.6.) In the setting of the lemma, a subgroup $H$ is diagonal if the following holds: There exist isomorphisms $\phi_{j, k}: G_{j} \rightarrow$ $G_{k}$ for some pairs of the groups $G_{1}, G_{2}, \ldots, G_{\ell}$. For each $k$, at most one $\phi_{j, k}$ should be chosen, and only when $j<k$. Then $H$ consists of those elements $\left(g_{1}, g_{2}, \ldots, g_{\ell}\right)$ such that $g_{k}$ is unrestricted when $\phi_{j, k}$ does not exist, and such that $g_{k}=\phi_{j, k}\left(g_{j}\right)$ when $\phi_{j, k}$ does exist.

After showing that various representations $V=X(\vec{c})$ of $\Gamma=\mathrm{PB}_{n}$ are dense, Lemma 3.5 is the main tool to show that they are jointly dense. Although it is stated in a different form, this tool is similar in spirit to the Independence Lemma of [1]. 
Lemma 3.6 (Stem) If $\pi: \widetilde{G} \rightarrow G$ is a stem extension of groups, and if $H \subseteq \widetilde{G}$ projects onto all of $G$, then $H=\widetilde{G}$.

One role of Lemma 3.6 is to clear up some confusion in the terminology for Lie groups. A surjective homomorphism $\pi: \widetilde{G} \rightarrow G$ is a stem extension if ker $\pi$ is central and lies in the commutator subgroup of $\widetilde{G}$. A group is quasisimple if it is a stem extension of a simple group. However, the standard terminology for Lie groups is different and slightly incompatible, as summarized in the following chart.

\begin{tabular}{c|c} 
groups & Lie groups \\
\hline simple & adjoint simple \\
quasisimple & simple
\end{tabular}

For example, $\operatorname{SL}(N, \mathbb{C})$ is only quasisimple as a group even though it is a simple Lie group. It is a stem extension of $\operatorname{PSL}(N, \mathbb{C})$, which is simple as a group and adjoint simple as a Lie group.

Lemma 3.7 (Commutator denseness) Consider the following diagram of groups:

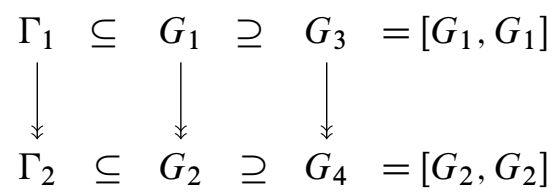

Suppose that each $G_{k}$ is a topological group, that the projection $G_{3} \rightarrow G_{4}$ is a stem extension that sends closed subgroups to closed subgroups, that the groups on the right are commutator subgroups as indicated, and that $\Gamma_{2}$ is the image of $\Gamma_{1}$ in $G_{2}$. If the closure of $\Gamma_{2}$ contains $G_{4}$, then $\Gamma_{1} \cap G_{3}$ is dense in $G_{3}$.

Note that the projection $G_{1} \rightarrow G_{2}$ in Lemma 3.7 need not be a stem extension. The lemma will be used when the four groups $G_{k}$ are $\operatorname{GL}(N, \mathbb{C}), \operatorname{PGL}(N, \mathbb{C}), \operatorname{SL}(N, \mathbb{C})$ and $\operatorname{PSL}(N, \mathbb{C})$; or when they are analogous real or unitary groups; or when they are products of these groups. It is useful in conjunction with Lemma 3.5 because, for instance, $\operatorname{PSL}(N, \mathbb{C})$ is an adjoint simple Lie group, while $\operatorname{SL}(N, \mathbb{C})$ is simple but not adjoint simple and $\operatorname{GL}(N, \mathbb{C})$ is not simple. Among other consequences, we need only be interested in representations up to projective equivalence.

Lemma 3.8 (Subrepresentation) Let $V$ be a representation of a group $G$ over a field $\mathbb{F}$, and suppose that $V$ decomposes as a direct sum of irreducible representations with multiplicity:

$$
V=n_{1} V_{1} \oplus n_{2} V_{2} \oplus \cdots \oplus n_{\ell} V_{\ell}
$$


Then every $G$-invariant subspace $W \subseteq V$ decomposes as

$$
W \cong m_{1} V_{1} \oplus m_{2} V_{2} \oplus \cdots \oplus m_{\ell} V_{\ell}
$$

where for each $k$,

$$
m_{k} V_{k} \cong W_{k} \subseteq n_{k} V_{k}
$$

Lemma 3.8 is used mainly for Lemma 3.9 below.

Lemma 3.9 (Connectedness) Suppose that a vector space $X$ over a field $\mathbb{F}$ is a multiplicity-free representation of two groups $G$ and $H$. Let

$$
X=V_{1} \oplus V_{2} \oplus \cdots \oplus V_{n}
$$

be the $G$-irreducible decomposition of $X$ and let

$$
X=W_{1} \oplus W_{2} \oplus \cdots \oplus W_{\ell}
$$

be the $H$-irreducible decomposition. Define a directed graph $C(X, G, H)$ on these summands, with an edge from $V_{j}$ to $W_{k}$ (or vice versa) if there exists $v \in V_{j}$ with a nonzero component in $W_{k}$. Then $X$ is an irreducible of the free product $G * H$ if and only if $C(X, G, H)$ is strongly connected.

To review, a directed graph $C$ is strongly connected if for every two vertices $p$ and $q$, there is a directed path in $C$ from $p$ to $q$.

We will use Lemma 3.9 in combination with Lemma 3.1 to inductively show that the adjoint space $V=\operatorname{sl}(X(n \cdot 1, c, t))$ is irreducible under $B_{n}$. The groups $G$ and $H$ will be two copies of $B_{n-1}$ in $B_{n}$.

Lemma 3.10 (Real Zariski) Let $G \subseteq \operatorname{SL}(N, \mathbb{C})$ be a subgroup which is real Zariski closed and complex Zariski dense. Then either $G=\operatorname{SL}(N, \mathbb{C})$, or the connected subgroup of $G$ is a conjugate of either $\operatorname{SL}(N, \mathbb{R})$ or $\operatorname{SU}(P, Q)$ with $P+Q=N$, or $\operatorname{SL}(N / 2, \mathbb{H})$ when $N$ is even. In particular, if the Lie algebra of $G$ contains $x$ and $i x$ for some $x$, then $G=\operatorname{SL}(N, \mathbb{C})$.

The first claim of Lemma 3.10 quickly implies cases 2 and 4 of Corollary 1.2 and real Zariski forms of cases 3 and 5. The second claim of Lemma 3.10 can be viewed as a complex version of Lemma 3.1.

Lemma 3.11 (Rotation) Let $\Gamma$ be a subgroup of a Lie group $G$ that acts on a vector space $V$. If $\Gamma$ has an elliptic element $g$ of infinite order, then $\Gamma$ is indiscrete in $G$ in the analytic topology. If $G=\operatorname{SU}(P, Q)$ with its defining representation and if $\Gamma$ is finitely generated and analytically dense, then $\Gamma$ has an elliptic element $g$ of infinite order. 
Lemma 3.11 is a tool to establish analytic denseness once the real Zariski closure has been computed.

Lemma 3.12 (Zassenhaus) If $\Gamma$ is a finitely generated discrete group and $G$ is a semisimple real Lie group, then the set of dense representations $\rho: \Gamma \rightarrow G$ (in the analytic topology) is an open subset of the set of all representations.

We will not directly use Lemma 3.12 as a tool, but it sheds light on what to expect in Theorem 1.4.

\section{Proofs of the tools and more lemmas}

In this section, we will prove the lemmas in Section 3. We will also discuss some other lemmas that are either related but not directly used, or are used but are more technical or secondary.

Lemma 3.1 holds in both the analytic topology and in the Zariski topology. It is a very useful folklore result in geometric group theory and yet its proof is elementary.

Proof of Lemma 3.1 The closure $\bar{\Gamma}$ is a $\Gamma$-invariant Lie group, so its Lie algebra $\mathfrak{c}$ is a $\Gamma$-invariant subalgebra of $\mathfrak{g}$. Since $\mathfrak{g}$ is $\Gamma$-irreducible, then either $\mathfrak{c}=\mathfrak{g}$, in which case $\bar{\Gamma}=G$, or $\mathfrak{c}=0$, in which case $\Gamma$ is discrete.

Lemma 3.2 is also elementary; it follows immediately from the fact that a linear subspace of $V$ is Zariski closed, and that the group action $G \times V \rightarrow V$ is Zariski continuous. What is somewhat less elementary is the context of other basic facts about the Zariski topology. For instance, one relevant fact is that the group law $G \times G \rightarrow G$ is Zariski continuous, ie, $G$ is a topological group relative to its Zariski topology. This follows from the fact that matrix multiplication is Zariski continuous, because there is a polynomial formula for matrix multiplication.

Lemma 3.3 is originally due to Chevalley and follows quickly from the StoneWeierstrass theorem; see Witte [9, Proposition 4.6.1].

Lemma 3.4 is a standard result in the theory of algebraic groups. It follows from another theorem of Chevalley, that the image of any algebraic set under an algebraic map is constructible; see Borel [2, Corollary 1.4].

Proof of Lemma 3.5 The proof is by induction on $\ell$. We first number the Lie factors of $G$ in order of nonincreasing dimension, and then after the Lie factors number the finite factors in order of nonincreasing cardinality. Let

$$
\pi: H \rightarrow G^{\prime}=G_{1} \times G_{2} \times \cdots \times G_{\ell-1}
$$


be the projection of $H$ onto the first $\ell-1$ factors. We can assume by induction that $\pi(H)=G^{\prime}$ by applying the lemma to $\pi(H)$ and then replacing $G^{\prime}$ by $\pi(H)$.

The kernel ker $\pi$ is a subgroup of $G_{\ell}$, and we claim that it is normal. Suppose that $h=(1,1, \ldots, 1, a) \in \operatorname{ker} \pi$. Since $H$ surjects onto $G_{\ell}$, this means that for every $g_{\ell} \in G_{\ell}$, there exists

$$
g=\left(g_{1}, g_{2}, \ldots, g_{\ell}\right) \in H
$$

for some choices of the other coordinates. Then

$$
g h g^{-1}=\left(1,1, \ldots, g_{\ell} a g_{\ell}^{-1}\right) \in H,
$$

so that ker $\pi$ is normal in $G_{\ell}$ as claimed. If $\operatorname{ker} \pi$ is nontrivial, then $\operatorname{ker} \pi=G_{\ell}$ and $H=G$ and we are done.

If instead ker $\pi=0$, then $H$ is the graph of a continuous group homomorphism $\alpha: G^{\prime} \rightarrow G_{\ell}$, and $\alpha$ factors as a direct product of homomorphisms $\alpha_{k}: G_{k} \rightarrow G_{\ell}$. The domain and target of $\alpha_{k}$ are both adjoint simple Lie groups and either $\operatorname{dim} G_{k} \geq \operatorname{dim} G_{\ell}$ or $\left|G_{k}\right| \geq\left|G_{\ell}\right|$. Therefore $\alpha_{k}$ is either the trivial homomorphism or an isomorphism. Moreover, since $G_{\ell}$ is noncommutative, it is not possible for more than one $\alpha_{k}$ to be surjective. Thus at most one $\alpha_{k}$ is an isomorphism and the others are the trivial homomorphism. This gives $H$ the structure promised by the lemma.

Proof of Lemma 3.6 Since $H$ projects onto $G$, it intersects every coset of $K=$ $\operatorname{ker} \pi$. Therefore it is enough to show that $H$ also contains $K$. By hypothesis, there exist commutators $[a, b] \in K$, with $a, b \in \widetilde{G}$, that generate $K$. Since $K$ is central, $[a, b]=[a g, b h]$ for any $g, h \in K$. Since $H$ meets every coset of $K$, we can choose $g, h$ so that $a g, b h \in H$. Thus $H$ contains $K$, as desired.

Proof of Lemma 3.7 The main idea is to define the commutator map

$$
c: G_{2} \times G_{2} \rightarrow G_{4} .
$$

This map is continuous and surjective, so it sends the dense set $\Gamma_{2} \times \Gamma_{2}$ to dense set in $G_{4}$, namely $\Gamma_{4}=\left[\Gamma_{2}, \Gamma_{2}\right]$. Then $\Gamma_{4}$ is covered by $\Gamma_{3}=\left[\Gamma_{1}, \Gamma_{1}\right]$. By Lemma 3.6, and by the hypothesis that the map from $G_{3}$ to $G_{4}$ sends closed subgroups to closed subgroups, $\Gamma_{3}$ is dense in $G_{3}$. At the same time, $\Gamma_{3} \subseteq \Gamma_{1}$, as desired.

Lemma 3.8 is a standard fact in representation theory. We sketch a proof for completeness, and because the lemma and proof are similar to Lemma 3.5 and its proof.

Proof of Lemma 3.8 We first rename the decomposition of $V$ as

$$
V=V_{1} \oplus V_{2} \oplus \cdots \oplus V_{\ell},
$$

where the summands are irreducible but not necessarily inequivalent. 
The proof is by induction on $\ell$. Let

$$
\pi: W \rightarrow V^{\prime}=V_{1} \oplus V_{2} \oplus \cdots \oplus V_{\ell-1}
$$

be the projection of $W$ onto the first $\ell-1$ summands. We can assume by induction that $\pi$ is surjective, by applying the lemma to $\pi(W)$ and replacing $V^{\prime}$ by $\pi(W)$. If $\operatorname{ker} \pi$ is nontrivial, it is a submodule of $V_{\ell}$ and it therefore is $V_{\ell}$. Thus if ker $\pi$ is nontrivial, then $W=V$ and we are done.

Suppose instead that $\pi$ is a bijection between $W$ and $V^{\prime}$. Then $W$ is the graph of a linear map $\alpha: V^{\prime} \rightarrow V_{\ell}$. The map $\alpha$ is the direct sum of maps $\alpha_{k}: V_{k} \rightarrow V_{\ell}$. By Schur's lemma, $\alpha_{k}=0$ when $V_{k} \not V_{\ell}$. (This part of Schur's lemma does not require an algebraically closed field.) Thus $\alpha$ is supported only on those summands of $V^{\prime}$ isomorphic to $V_{\ell}$, which is the structure promised by the lemma.

Proof of Lemma 3.9 The idea of this lemma is that if several assertions are related by a strongly connected set of inferences, then the assertions are all equivalent. For instance it is common to say " $p$ implies $q$ implies $r$ implies $p$, therefore $p, q$ and $r$ are equivalent".

Let $Y$ be a subspace of $X$ which is invariant under both $A$ and $B$, and consider the assertions that $V_{j} \subseteq Y$ or that $W_{k} \subseteq Y$. When $C(X, G, H)$ has an edge from $V_{j}$ to $W_{k}$, then $V_{j} \subseteq Y$ implies $W_{k} \subseteq Y$, and vice-versa. By hypothesis, these implications are a strongly connected graph, so that $Y$ must either contain all of the summand or none of them. Thus $Y=X$ or $Y=\{0\}$, so that $X$ is irreducible.

On the other hand, if $C(X, G, H)$ is not strongly connected, then it has a strongly connected component $D$ with no outward edges. It is easy to confirm that the summands $\left\{V_{j}\right\}$ of $D$ have the same direct sum as the summands $\left\{W_{k}\right\}$ of $D$. This direct sum $Y$ is then both $G$-invariant and $H$-invariant, so that $X$ is not irreducible.

Proof of Lemma 3.10 This lemma is almost just the classification of real forms of the complex simple Lie algebra $\operatorname{sl}(N, \mathbb{C})$, but it needs some extra reasoning at the level of algebraic groups. The real forms are well-known and match the conclusion of the lemma; see for instance Fulton and Harris [5, Section 26.1] for a list at the Lie algebra level.

The real algebraic group $G$ has a complexification $G_{\mathbb{C}}$. (If $G$ is already complex, then $G_{\mathbb{C}} \cong G \times G$.) We can assume, by passing to a finite-index subgroup, that $G$ is connected in the real Zariski topology. Then by construction, $G_{\mathbb{C}}$ is a subgroup of the complexification $\operatorname{SL}(N, \mathbb{C}) \times \operatorname{SL}(N, \mathbb{C})$ of $\operatorname{SL}(N, \mathbb{C})$ as a real algebraic group. $G_{\mathbb{C}}$ maps to each factor of $\operatorname{SL}(N, \mathbb{C})$, and each of these maps $\alpha$ and $\bar{\alpha}$ extends the inclusion 
of $G$. The map $\alpha$ is complex algebraic and $\bar{\alpha}$ is its complex conjugate. By Lemma 3.4, the image of $\alpha$ is closed, and then surjective by the hypothesis that $G$ is complex Zariski dense. Thus by Lemma 3.5, $G_{\mathbb{C}}$ is either all of $\operatorname{SL}(N, \mathbb{C}) \times \operatorname{SL}(N, \mathbb{C})$, or it is the graph of a real algebraic automorphism of $\operatorname{SL}(N, \mathbb{C})$. Thus either $G=\operatorname{SL}(N, \mathbb{C})$, or $G_{\mathbb{C}}=\operatorname{SL}(N, \mathbb{C})$ and $G$ is a real form, as desired.

Proof of Lemma 3.11 The first claim of the lemma is elementary. In a basis in which $g$ is diagonal, it is contained in the compact group of diagonal unitary matrices. Therefore if $g$ has infinite order, it generates an indiscrete subgroup.

The second claim rests on two ideas. The first idea is to argue by counting degrees of freedom that the elliptic elements in $\operatorname{SU}(P, Q)$ include an open set. Therefore any dense subgroup has many elliptic elements. Let $N=P+Q$. Then

$$
\operatorname{dim} \operatorname{SU}(P, Q)=N^{2}-1 .
$$

Moreover, the set of orthogonal line bases in $\mathbb{C}^{(P, Q)}$, with $P$ positive lines and $Q$ negative lines, is a manifold of complex dimension $\left(\begin{array}{c}N \\ 2\end{array}\right)$ and real dimension $N^{2}-N$. If $B$ is such a line basis, then we can construct an elliptic element $g$ which is diagonal in this basis. The element $g$ has $N-1$ additional degrees of freedom, because its entries are complex numbers of norm 1 whose product is 1 . Indeed, for every $\epsilon>0$, there is an open set of elliptic elements with at least one eigenvalue $\exp (i \theta)$ with $\epsilon<\theta<2 \epsilon$. Thus $\Gamma$ must have elliptic elements of either infinite order or unbounded finite order.

The second idea is similar to the proof of Malcev's theorem that $\Gamma$ is residually finite. The matrix entries of the elements of $\Gamma$ lie in a field $\mathbb{F} \subseteq \mathbb{C}$ which is finitely generated over $\mathbb{Q}$. It is an interesting fact that any subfield of a finitely generated field is still finitely generated [10, Theorem 3.3.5]; in particular the algebraic subfield of $\mathbb{F}$ is a finite-dimensional field $\mathbb{F}^{\prime} \supseteq \mathbb{Q}$. A root $\lambda$ of a characteristic polynomial of an element $g \in G$ is then either transcendental, or it lies in a field $\mathbb{F}^{\prime \prime} \supseteq \mathbb{F}^{\prime}$ whose degree over $\mathbb{F}^{\prime}$ is bounded by $N$. This imposes an upper bound on the order of $\lambda$ if it is a root of unity, because for every $n$, there are only finitely many roots of unity of algebraic degree $n$. Thus it is not possible for $\Gamma$ to have elliptic elements of unbounded finite order; it must instead have elliptic elements of infinite order.

Proof of Lemma 3.12 It is a folklore result, with a careful proof given by Breuillard and Gelander [3, Theorem 2.1], that if $\Gamma$ has elements $g_{1}, \ldots, g_{m}$ in a small enough neighborhood $U$ of $1 \in G$ such that $\log \left(g_{1}\right), \ldots, \log \left(g_{m}\right)$ generate the Lie algebra $\mathfrak{g}$, then $\Gamma$ is dense. (We assume a neighborhood $0 \in V \subseteq \mathfrak{g}$ such that the exponential map exp: $\mathfrak{g} \rightarrow G$ is injective on $V$. Thus, logarithms are well-defined on any open subset of $\exp (V)$.) This result is related to a theorem of Zassenhaus and Kazhdan-Margulis: 
If $\Gamma$ is a discrete subgroup of $G$, then for a suitable $U$ independent of $\Gamma, \Gamma \cap U$ is contained in a connected, nilpotent Lie subgroup of $G$.

Let us be precise about the topology on the space of representations. If $\Gamma$ is generated by a finite set $A$, then the representation variety $\{\rho: \Gamma \rightarrow G\}$ is a closed subset of the set of all functions $f: A \rightarrow G$. Moreover, the induced topology is independent of the generating set, since the topology does not change if we enlarge $A$.

If $\rho: \Gamma \rightarrow G$ is a dense representation, then we can find elements

$$
a_{1}, a_{2}, \ldots, a_{m} \in \Gamma
$$

such that $\rho\left(a_{k}\right) \in U$, and such that

$$
\log \left(\rho\left(a_{1}\right)\right), \log \left(\rho\left(a_{2}\right)\right), \ldots, \log \left(\rho\left(a_{m}\right)\right)
$$

span $\mathfrak{g}$. Spanning $\mathfrak{g}$ is an open condition, since $a \times b$ matrices of full rank are an open subset of all $a \times b$ matrices. Thus, if $\rho^{\prime}$ is close enough to $\rho$, then

$$
\log \left(\rho^{\prime}\left(a_{1}\right)\right), \log \left(\rho^{\prime}\left(a_{2}\right)\right), \ldots, \log \left(\rho^{\prime}\left(a_{m}\right)\right)
$$

still span $\mathfrak{g}$. Since they span $\mathfrak{g}$, they generate $\mathfrak{g}$ and thus $\rho^{\prime}$ is dense.

\section{Quantum algebra}

In this section we review some of the properties of the Kauffman bracket and by extension the Jones polynomial. For a more complete introduction to this theory, see for instance Kauffman and Lins [7].

The Jones-Wenzl projector of color $c$ can be defined recursively as follows:

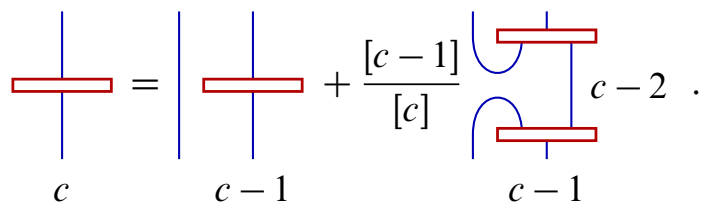

Here and below, a strand labelled by $n$ is shorthand for $n$ strands. Also

$$
[n]=\frac{t^{n / 2}-t^{-n / 2}}{t^{1 / 2}-t^{-1 / 2}}
$$

is a quantum integer. Because of a quantum integer appearance in the denominator, if $t$ has order $r$, then the Jones-Wenzl projector of color $c$ is only defined when $c \leq r-1$. Moreover, the color $r-1$ is suppressed, because any reduced skein space such as $X(n \cdot 1, r-1, t)$ vanishes. So we say that $c$ is an admissible color if $c \leq r-2$. 
If $\vec{c}=\left(c_{1}, c_{2}, \ldots, c_{\ell}\right)$ is a vector of admissible colors, then we can define a generalized skein space $W(\vec{c}, t)$ and reduced skein space $X(\vec{c}, t)$. The space $W(\vec{c}, t)$ is defined using $\ell$ clasps of the respective colors:

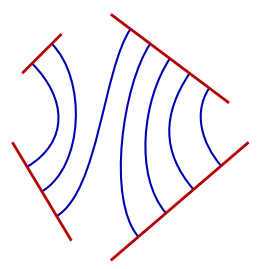

As before, the reduced space $X(\vec{c}, t)$ is defined by gluing together two skeins with projectors and dividing by the kernel of the resulting bilinear form.

We will also need three standard results in the skein theory of the Kauffman bracket and one structural result. We omit the proofs; see [7] for the general theory.

The structural result that we will need is the following well-known splitting formula.

Theorem 5.1 (Splitting) If $\vec{c}$ and $\vec{c}^{\prime}$ are two lists of colors and $\vec{c} \oplus \vec{c}^{\prime}$ is their concatenation, then

$$
X\left(\vec{c} \oplus \vec{c}^{\prime}, t\right) \cong \bigoplus_{a} X(\vec{c}, a, t) \otimes X\left(a, \vec{c}^{\prime}, t\right),
$$

where the map

$$
X(\vec{c}, a, t) \otimes X\left(a, \vec{c}^{\prime}, t\right) \rightarrow X\left(\vec{c} \oplus \vec{c}^{\prime}, t\right)
$$

is given by gluing together skeins using a projector of weight $a$.

We will need the effect of a full twist on a projector.

\section{Lemma 5.2}

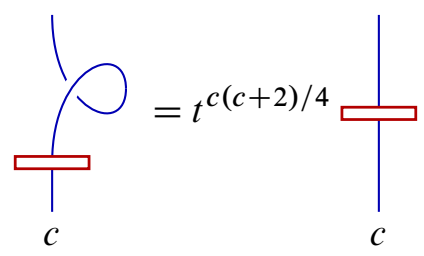

We will also want an explicit change-of-basis formula between the two ways to split $X(c, 1, c, 1, t)$. 


\section{Lemma 5.3 Let}
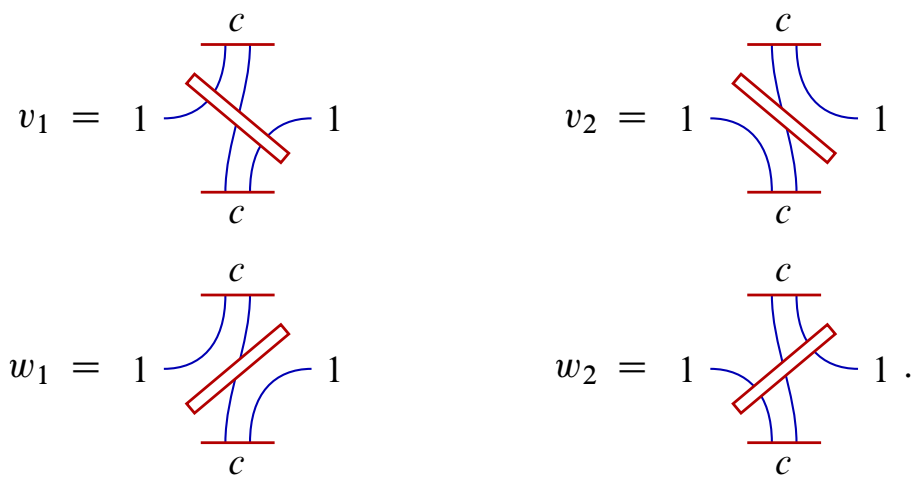

Then

$$
\begin{aligned}
& v_{1}=\frac{[c][c+2]}{[c+1]^{2}} w_{1}+\frac{1}{[c+1]} w_{2} \\
& v_{2}=-\frac{1}{[c+1]} w_{1}+w_{2} .
\end{aligned}
$$

Finally we will need the following two dimension inequalities:

Lemma 5.4 Let $t$ be a root of unity of order $r$ with $r$ even, and let $0 \leq c<(r-2) / 2$. If $\operatorname{dim} X(n \cdot 1, c, t)>0$, then

$$
\operatorname{dim} X(n \cdot 1, c, t)>\operatorname{dim} X(n \cdot 1, r-2-c, t) .
$$

Proof Theorem 5.1 implies a recursive characterization of the numbers

$$
d(n, c, r)=\operatorname{dim} X(n \cdot 1, c, t)
$$

when $c$ is admissible. Namely,

$$
\begin{aligned}
d(0,0, r) & =1 & & \\
d(0, c, r) & =0 & & (0<c) \\
d(n, c, r) & =d(n-1, c-1, r)+d(n-1, c+1, r) & & (0<c<r-2) \\
d(n, 0, r) & =d(n-1,1, r) & & \\
d(n, r-2, r) & =d(n-1, r-3, r) . & &
\end{aligned}
$$

A simple induction argument shows that $d(n, c, r)>0$ when $c \leq n$ and $c+n$ is even and that $d(n, c, r)=0$ otherwise. Now let

$$
e(n, c, r)=d(n, c, r)-d(n, r-2-c, r) .
$$

It is easy to check that $e(n, c, r)$ satisfies the same recurrence as $d(n, c, r / 2)$. Therefore these numbers are equal, and the inequality for $d(n, c, r)$ is the desired claim. 
Lemma 5.5 If $1 \leq \operatorname{dim} X(n \cdot 1, c, t) \leq 2$ and $c \geq 5$, then either $c=n$ or $n \leq 3$ or $(n, c)=(0,4)$.

Proof We use the abbreviation $d(n, c, r)$ in Lemma 5.4 and the recurrence that these numbers satisfy. We obtain

$$
\begin{array}{rlrl}
d(4,0, r) & =2 & d(4,2, r) & =3 \\
d(5,1, r) & =5 & 3 \leq d(5,3, r) & \leq 4 .
\end{array}
$$

It is then easy to check the lemma by induction for $n \geq 6$.

\section{Proof of the main results}

The proof of Theorem 1.1 is by mutual induction with Theorem 1.3. For convenience, we let $r$ be the order of $t$ if it is a root of unity, and let $r=\infty$ otherwise.

Proof of Theorem 1.3 Lemma 3.7 tells us that we can replace each group $G_{k}$ with $\operatorname{PSL}(X), \operatorname{PSL}\left(X_{\mathbb{R}}\right)$, or $\operatorname{PSU}(X)$, where $X=X(n \cdot 1, c, t)$. Lemma 3.4 tells us that we can replace the action of $B_{n}$ by its Zariski closure in each factor. Then Lemma 3.5 tells us that our aim is to show that two representations $X=X(n \cdot 1, c, t)$ and $X^{\prime}=X\left(n \cdot 1, c^{\prime}, t\right)$ are inequivalent, in the sense that there does not exist an isomorphism $\alpha$ of the corresponding groups that equates the actions of $B_{n}$. Moreover, the graph of $\alpha$ has to be closed in the relevant Zariski topology, which implies that $\alpha$ is continuous in the analytic topology.

Consider first the case

$$
\alpha: \operatorname{PSL}(X) \rightarrow \operatorname{PSL}\left(X^{\prime}\right) .
$$

In this case, the only associated automorphisms of $\operatorname{PGL}(N, \mathbb{C})$ are

$$
\begin{array}{ll}
\alpha(x)=x & \alpha(x)=\left(x^{-1}\right)^{T} \\
\alpha(x)=\bar{x} & \alpha(x)=\left(\bar{x}^{-1}\right)^{T} .
\end{array}
$$

Here $x$ is a matrix. If instead $\alpha$ is a map from $\operatorname{PSU}(X)$ to $\operatorname{PSU}\left(X^{\prime}\right)$, or from $\operatorname{PSL}\left(X_{\mathbb{R}}\right)$ to $\operatorname{PSL}\left(X_{\mathbb{R}}^{\prime}\right)$, then the automorphisms have the same formulas, except that the last two are equivalent to the first two. In the complex Zariski topology, only the first two choices of $\alpha$ have a closed graph. Finally, since we are working in projective linear groups, $x$ is only defined up to a scalar factor.

So we are interested in a "fingerprint" of the representation $X$ that will distinguish it from other representations $X^{\prime}$. The fingerprint can make use of the spectrum of the action of an element $g \in B_{n}$, up to a scalar factor and up to inversion, because that 
information is preserved by all choices of $\alpha$. If $\operatorname{dim} X=1$, then fingerprinting is not necessary because the corresponding group such as $\operatorname{PSL}(X)$ is trivial.

By Theorem 5.1,

$$
X(n \cdot 1, c, t) \cong X((n-1) \cdot 1, c+1, t) \oplus X((n+1) \cdot 1, c-1, t) .
$$

Let $g$ be the full twist on the first $n-1$ strands. Then by Lemma 5.2, its eigenvalues are proportional to $t^{(c+1)(c+3) / 4}$ on $X((n-1) \cdot 1, c+1, t)$ and $t^{(c-1)(c+1) / 4}$ on $X((n-1) \cdot 1, c-1, t)$. Or, after rescaling, the eigenvalues are $t^{c+1}$ and 1 . One of the eigenvalues is suppressed when $c=0$ and when $c=r-2$. Together with $n$ itself, this is a complete fingerprint for $X$ when $t$ is not a root of unity or when $r$ is odd. When $r$ is even, this data does not distinguish $c$ from $r-2-c$, because we can switch the spectrum $\left(t^{c+1}, 1\right)$ with $\left(t^{r-2-c+1}, 1\right)$ by inverting $t$ and rescaling. However, by Lemma 5.4,

$$
\operatorname{dim} X(n \cdot 1, c, t)>\operatorname{dim} X(n \cdot 1, r-2-c, t) .
$$

Thus the strand number $n$, the eigenvalues of $g$, and the dimension $\operatorname{dim} X$ are a complete fingerprint for $X$ and prevent the existence of the isomorphism $\alpha$.

Proof of Theorem 1.1 The proof is by induction and the base case is $X(3 \cdot 1,1, t)=$ $X(4 \cdot 1,0, t)$. First suppose that $t$ is not a root of unity. In this case, let $\tau_{1}$ and $\tau_{2}$ be the braid generators of $B_{3}$ acting on $X(3 \cdot 1,1, t)$. Then the action of $\tau_{1}$ is

$$
\left(\begin{array}{cc}
t^{-3 / 4} & 0 \\
0 & -t^{1 / 4}
\end{array}\right)
$$

in the basis
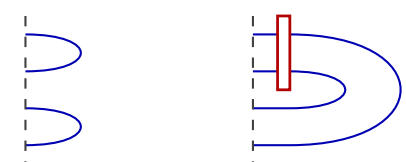

while the action of $\tau_{2}$ has the same matrix as $\tau_{1}$, but in the basis
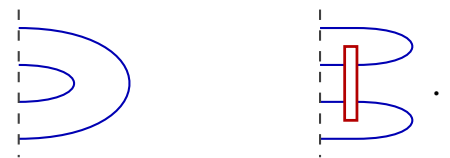

By Lemma 5.3, the change of basis matrix is

$$
M=\left(\begin{array}{cc}
-1 /[2] & 1 \\
{[3] /[2]^{2}} & 1 /[2]
\end{array}\right)
$$


The Zariski closure in $\operatorname{PSL}(2, \mathbb{C})$ of the action of $\tau_{1}$, in its basis, is

$$
\overline{\left\langle\tau_{1}\right\rangle}=\left\{\left(\begin{array}{cc}
z & 0 \\
0 & z^{-1}
\end{array}\right)\right\}_{z \in \mathbb{C} \backslash\{0\}} .
$$

The Lie algebra $\operatorname{sl}(2, \mathbb{C})$ has a multiplicity-free decomposition under this action, and by Lemma 3.8 it has only four $\tau_{1}$-invariant subspaces that contain the Lie algebra of $\overline{\left\langle\tau_{1}\right\rangle}$ :

$$
\left(\begin{array}{ll}
* & 0 \\
0 & *
\end{array}\right) \quad\left(\begin{array}{ll}
* & * \\
0 & *
\end{array}\right) \quad\left(\begin{array}{ll}
* & 0 \\
* & *
\end{array}\right) \quad\left(\begin{array}{ll}
* & * \\
* & *
\end{array}\right) .
$$

Because the change-of-basis matrix $M$ is full, the Lie algebra of the $\overline{\left\langle\tau_{2}\right\rangle}$ only lies in the last choice. (This argument is actually a special case of Lemma 3.9.) Thus the Lie algebra of the Zariski closure is all of $\operatorname{sl}(2, \mathbb{C})$, and the group is $\operatorname{PSL}(2, \mathbb{C})$.

Suppose that $t$ is a root of unity. For convenience we instead consider the action of $B_{4}$ on $X=X(4 \cdot 1,0, t)$. Also for convenience, we assume that $t$ is a principal root of unity, which is not a loss of generality because the complex Zariski topology is Galois-invariant. Let $\tau_{1}, \tau_{2}, \tau_{3}$ be the braid generators, and let

$$
\sigma_{3}=\tau_{2} \tau_{1} \quad \sigma_{4}=\tau_{3} \tau_{2} \tau_{1} .
$$

Then in $\mathrm{PU}(X) \cong \operatorname{PSU}(X) \cong \mathrm{SO}(3), \sigma_{3}$ has order 3 and $\sigma_{4}$ has order 2. Meanwhile

$$
\tau_{3}=\sigma_{4} \sigma_{3}^{-1}
$$

has eigenvalue ratio $-t$, so it has order $s=2 r$ when $r$ is odd, $s=r / 2$ when $r=4 k+2$, and $s=r$ when $r=4 k$. The braids $\sigma_{3}, \sigma_{4}$, and $\tau_{3}$ generate $B_{4}$, and their action, if finite, is that of the $(2,3, s)$ triangle group. However, this triangle group is infinite when $s \geq 7$, so the action is dense. (It is also infinite when $s=6$, but this implies that $r=3$, which is excluded.)

When $r=10$, then $s=5$, and the action of $B_{4}$ is that of the icosahedral group. Crucially, this action is adjoint-irreducible and the image is a simple group. These properties will make it usable as a base for induction even though it is not a case of the theorem. The inductive step of this case will be saved for last.

For the inductive step, let $X=X(n \cdot 1, c, t)$. If $c=n$, there is nothing to prove because $\operatorname{dim} X=1$. If $c=0$ and $n>0$, there is also nothing to do because

$$
X(n \cdot 1,0, t)=X((n-1) \cdot 1,1, t) .
$$

Likewise if $t$ has order $r$ and $c=r-2$, then

$$
X(n \cdot 1, c, t)=X((n-1) \cdot 1, c-1, t) .
$$


So suppose that $n>c>0$ and that either $t$ is not a root of unity or that $c \leq r-3$. Then as in the proof of Theorem 1.3,

$$
X(n \cdot 1, c, t) \cong X((n-1) \cdot 1, c+1, t) \oplus X((n+1) \cdot 1, c-1, t) .
$$

Moreover, there are two different splittings, depending on whether we restrict to the first $n-1$ strands or the last $n-1$ strands. Let $G$ be the braid group on the first $n-1$ strands and let $H$ be the braid group on the last $n-1$ strands. Let $V_{1}$ and $V_{2}$ be the $G$-invariant summands with colors $c+1$ and $c-1$, and likewise let $W_{1}$ and $W_{2}$ be the $H$-invariant summands.

We want to determine the position of $V_{1}$ and $V_{2}$ relative to $W_{1}$ and $W_{2}$ using the splitting

$$
X \cong \bigoplus_{a} X((n-2) \cdot 1, a, t) \otimes X(a, 1, c, 1),
$$

where the first factor uses the middle $n-2$ strands. In particular we will use the summand with $a=c$, which must be nonzero given our assumptions on $n$. Call this summand $Y \otimes Z$, where

$$
Y=X((n-2) \cdot 1, c, t) \quad Z=X(c, 1, c, 1) .
$$

Then the four skeins in Lemma 5.3 are in $Z$; when tensored with $Y$, they place copies of $Y$ in each of $V_{1}, V_{2}, W_{1}, W_{2}$. Lemma 5.3 then says that the change-of-basis matrix between these skeins has no zero entries.

Thus the graph $C(X, G, H)$ of Lemma 3.9 is strongly connected, so that $X$ is an irreducible representation of $B_{n}$. However, we are interested in $\operatorname{sl}(X)$, which is a more complicated case. This vector space decomposes as

$$
\operatorname{sl}(X) \cong \operatorname{sl}\left(V_{1}\right) \oplus \operatorname{sl}\left(V_{2}\right) \oplus\left(V_{1} \otimes V_{2}^{*}\right) \oplus\left(V_{2} \otimes V_{1}^{*}\right) \oplus I_{G}
$$

where the last summand $I_{G}$ corresponds to traceless operators on $X$ that act by scalars on both $V_{1}$ and $V_{2}$. By induction, and by Lemma 3.2, each term in this decomposition is $G$-irreducible, but we would also like to know that it is multiplicity-free. For that purpose, Theorem 1.3 says that we can use the action of $\operatorname{SL}\left(V_{1}\right) \times \operatorname{SL}\left(V_{2}\right)$ to distinguish the summands. Note also that if $\operatorname{dim} V_{k}=1$, then $\operatorname{sl}\left(V_{k}\right)$ vanishes; it is a null term in the decomposition.

Recall that the adjoint representation of $\operatorname{SL}(V)$ is irreducible and inequivalent to the defining representation, and that the defining representation is not self-dual if $\operatorname{dim} V \geq 3$. Thus all of the terms in the decomposition are inequivalent, except that $V_{1} \otimes V_{2}^{*}$ and $V_{2} \otimes V_{1}^{*}$ are equivalent when $V_{1}$ and $V_{2}$ have dimension at most 2. By Lemma 5.5, the exception occurs only when $n=4$ and $c=2$. 
To address this exception, let $g \in G$ be the full twist on the first $n-1$ strands, as in the proof of Theorem 1.3. The eigenvalue ratio of $g$ acting on $V_{1}$ and $V_{2}$ is $t^{c+1}=t^{3}$. Therefore its eigenvalue ratio on $V_{1} \otimes V_{2}^{*}$ and $V_{2} \otimes V_{1}^{*}$ is $t^{6}$. We know that $t^{6} \neq 1$ since $t$ is a nonlattice root of unity, so $V_{1} \otimes V_{2}^{*}$ and $V_{2} \otimes V_{1}^{*}$ are distinguished by the action of $g$. Thus Equation (2) is a multiplicity-free decomposition in all germane cases.

By the same reasoning,

$$
\operatorname{sl}(X) \cong \operatorname{sl}\left(V_{1}\right) \oplus \operatorname{sl}\left(V_{2}\right) \oplus\left(V_{1} \otimes V_{2}^{*}\right) \oplus\left(V_{2} \otimes V_{1}^{*}\right) \oplus I_{H}
$$

is the $H$-irreducible decomposition of $\operatorname{sl}(X)$ and it is also multiplicity-free. Thus Lemma 3.9 applies to $\operatorname{sl}(X)$, provided that $C(\operatorname{sl}(X), G, H)$ is strongly connected. This is our final claim to establish the theorem.

Let $v_{1} \otimes v_{2}^{*} \in V_{1} \otimes V_{2}^{*}$ be a vector, and consider its $H$-decomposition

$$
v_{1} \otimes v_{2}^{*}=w_{1} \otimes w_{1}^{*}+w_{1} \otimes w_{2}^{*}+w_{2} \otimes w_{1}^{*}+w_{2} \otimes w_{2}^{*} .
$$

By the structure of $C(X, G, H)$, the vector $v_{1}$ and the dual vector $v_{2}^{*}$ can be chosen so that all four terms in the $H$-decomposition are nonzero. Moreover, $w_{k} \otimes w_{k}^{*}$ must have a nonzero component in $\operatorname{sl}\left(V_{k}\right)$ when $\operatorname{dim} V_{k} \geq 2$, because it is a rank 1 operator and cannot be proportional to the identity. Thus in $C(\operatorname{sl}(X), G, H)$, there is an edge from $V_{1} \otimes V_{2}^{*}$ to every $H$-invariant summand other than possibly $I_{H}$.

We also claim that there is an edge from $I_{G}$ to at least one $H$-irreducible summand other than $I_{H}$. This will happen unless $I_{G}=I_{H}$. They cannot be equal, because the eigenspaces of $x \in I_{G}$ are $V_{1}$ and $V_{2}$, while the eigenspaces of $y \in I_{H}$ are $W_{1}$ and $W_{2}$; and these subspaces of $X$ are different.

Moreover, $\operatorname{sl}(X)$ is a self-dual representation using the bilinear form $\operatorname{Tr}(x y)$ (the Killing form). This implies that if $C(\operatorname{sl}(X), G, H)$ has an edge from a vertex $A$ to a vertex $B$, it also has an edge from $B^{*}$ to $A^{*}$. Moreover, for all of the edges constructed so far, we can switch $V_{1}$ and $V_{2}$ with $W_{1}$ and $W_{2}$. All told, these edges render $C(\operatorname{sl}(X), G, H)$ strongly connected. This concludes the proof when $r \neq 10$.

Finally let $r=10$. The projective action of $B_{3}$ on $X(3 \cdot 1,1, t)$ is that of the icosahedral group. Since this action is both adjoint-irreducible and a finite simple group, it can be used in Theorem 1.3, and in the rest of the above argument when $n=4$. We can conclude that the action of $B_{4}$ on $X(4 \cdot 1,2, t)$ is adjoint-irreducible, but we also claim that it is indiscrete, and this claim needs a separate argument. An ad hoc search in Sage reveals that commutator

$$
g=\left[\tau_{2}, \tau_{2} \tau_{3}^{3} \tau_{2} \tau_{1}^{-1}\right]
$$


\# Set up rings and variables.

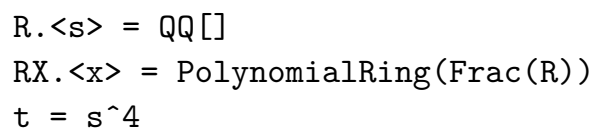

\section{\# Conveniences}

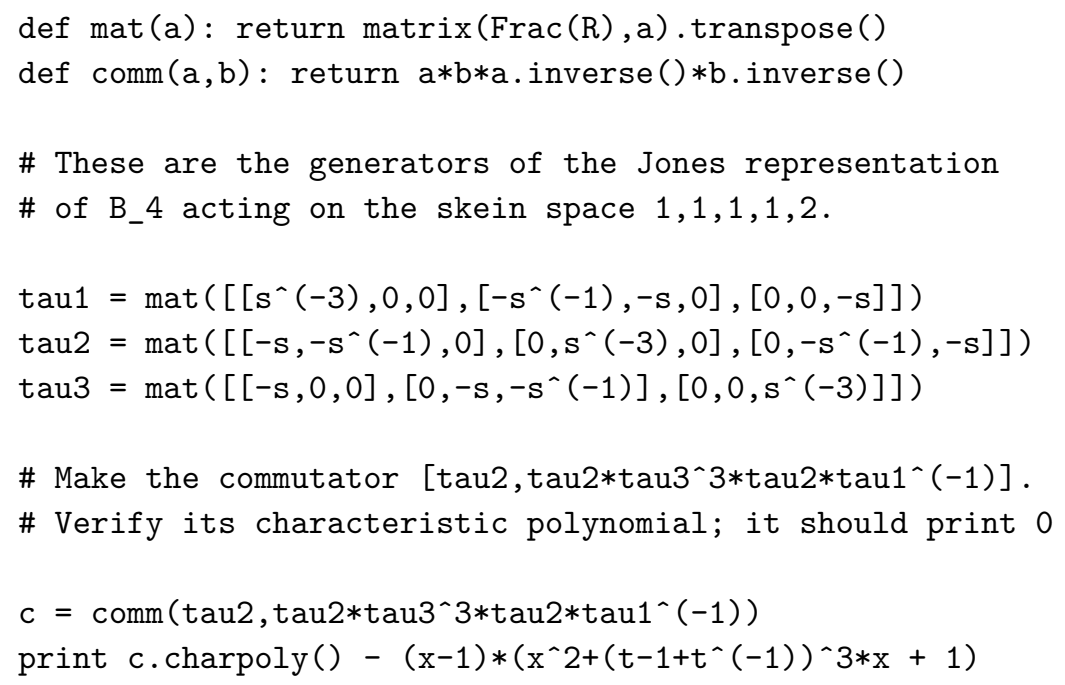

Figure 1: Sage code to compute $\left[\tau_{2}, \tau_{2} \tau_{3}^{3} \tau_{2} \tau_{1}^{-1}\right]$

has characteristic polynomial

$$
\chi_{g}(x)=(x-1)\left(x^{2}+\left(t-1+t^{-1}\right)^{3} x+1\right)
$$

in its action on $X(4 \cdot 1,2, t)$, for any $t$ for which this space is 3 -dimensional. (See Figure 1.) When $t=\exp (\pi i / 5)$, the roots $\lambda$ other than 1 have the form $\exp (i \theta)$ with

$$
|\theta| \approx 96.778652^{\circ} \text {. }
$$

We claim that this is an irrational angle, although at first glance it is not clear. By Galois theory, $\lambda$ has degree at most 4 over $\mathbb{Q}$. Thus if $\lambda$ did have finite order $s$, the Euler totient $\phi(s)$ of $s$ would be at most 4 , so that $s \leq 10$. The approximation to $\theta$ thus tells us that $g$ has infinite order and the action of $B_{4}$ is dense.

The projective action of $B_{4}$ on $X(4 \cdot 1,0, t)$ is also that of the icosahedral group. However, this and the action on $X(4 \cdot 1,2, t)$ are enough to show adjoint irreducibility 
when $n=5$. When $n=5$ none of the actions can be discrete, and the case $r=10$ thus merges with the other cases.

Proof of Corollary 1.2 As suggested in Section 3, cases 2 and 4 follow from Lemma 3.10 together with the existence of $X(n \cdot 1, c, t)_{\mathbb{R}}$ when $t$ is real, and the invariant Hermitian structure on $X(n \cdot 1, c, t)$ when $|t|=1$ as described in Section 5. Lemma 3.10 likewise implies cases 3 and 5 follow in the real Zariski topology.

To complete cases 3 and 5, note that the criterion of Lemma 3.11 is easily satisfied, albeit for different reasons. In case 3 , the ratio of the eigenvalues of a braid generator $\tau_{1}$ (say) is $-t$. Thus when $|t|=1$ but $t$ is not a root of unity, then $\tau_{1}$ is an elliptic element of infinite order. In case 5, if $B_{n}$ acts discretely, then it acts finitely and is not complex Zariski dense in $\operatorname{SL}(X(n \cdot 1, c, t))$. As mentioned, case 5 also follows from Lemma 3.3.

As mentioned, case 6 follows from Lemma 3.1.

Finally consider case 1 . This case is established by induction on $n$. First, let $X=$ $X(3 \cdot 1,1, t)$. By Lemma 3.10, we wish to show that the projective action of $B_{3}$ is not contained in any copy of $\operatorname{PSU}(2)$ or $\operatorname{PSU}(1,1)$ or $\operatorname{PSL}(2, \mathbb{R})$. Specially to dimension 2 , $\operatorname{PSU}(1,1)$ and $\operatorname{PSL}(2, \mathbb{R})$ are conjugate in $\operatorname{PSL}(2, \mathbb{C})$. Moreover, the normalizer of $\operatorname{PSL}(2, \mathbb{R})$ can be written as $\operatorname{PGL}(2, \mathbb{R})$ in $\operatorname{PGL}(2, \mathbb{C})=\operatorname{PSL}(2, \mathbb{C})$. The normalizer of PSU(2) is itself.

Again, the eigenvalue ratio of the braid generator $\tau_{1}$ is $-t$. Since the ratio does not have norm 1, it is not contained in any conjugate of PSU(2). Since the ratio is not real, it is not contained in any conjugate of $\operatorname{PGL}(2, \mathbb{C})$.

In the inductive case, let $X=X(n \cdot 1, c, t)$. This $X$ is a direct sum of many copies of $X(3 \cdot 1,1, t)$ and $X(3 \cdot 1,3, t)$. The commutator subgroup $\left[B_{3}, B_{3}\right]$ of $B_{3}$ must still be real Zariski dense in its action on $X(3 \cdot 1,1, t)$, while its action on $X(3 \cdot 1,3, t)$ is trivial. Therefore the Lie algebra of the real Zariski closure of the action of $\left[B_{3}, B_{3}\right]$ includes both $x$ and $i x$ for some $x \in \operatorname{sl}(X)$. By Lemma 3.10, this implies that the action of $B_{n}$ is real Zariski dense.

\section{Other results}

Proof of Theorem 1.4 It will be more convenient to consider the action of $B_{4}$ on $X(4 \cdot 1,0, t)$. Case 2 is a special case of Theorem 1.1 , given that $\operatorname{SU}(X(4 \cdot 1,0, t))$ is compact.

To analyze cases 1 and 3, it is useful to work in

$$
\operatorname{PGL}(2, \mathbb{R}) \subseteq \operatorname{PGL}(2, \mathbb{C})=\operatorname{PSL}(2, \mathbb{C})
$$




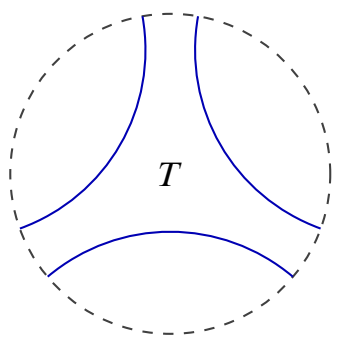

(a) Hyperideal triangle

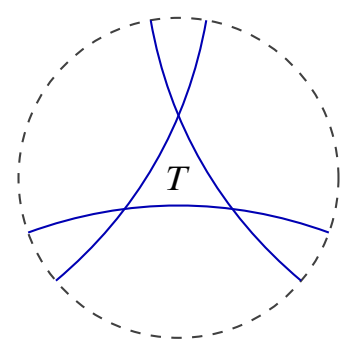

(b) Nonideal triangle

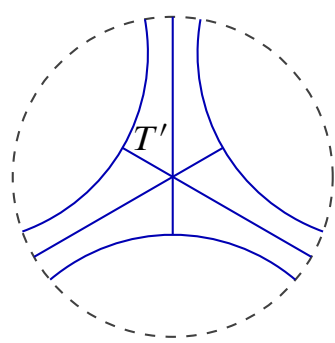

(c) Barycentric subdivision

Figure 2: Hyperbolic triangles

and to recall that $\operatorname{PGL}(2, \mathbb{R})$ is the isometry group of the hyperbolic plane $\mathbb{H}^{2}$. (Note that in Lemma 3.10, " $\mathbb{H}$ " refers instead to the quaternions.) The nontrivial elements with positive determinant are rotations, hyperbolic translations, and parabolic motions. The elements with negative determinant are reflections and glide reflections. Recall also that $\operatorname{PSL}(2, \mathbb{C})$ is the rotation group of hyperbolic space $\mathbb{H}^{3}$. Reflections in $\operatorname{PGL}(2, \mathbb{R})$ are realized as rotations in $\mathbb{H}^{3}$ that flip over $\mathbb{H}^{2}$. In particular, reflections are conjugate in $\operatorname{PSL}(2, \mathbb{C})$ to rotations by $\pi$, even though they are not conjugate in $\operatorname{PGL}(2, \mathbb{R})$.

An element $g$ can be analyzed in terms of its eigenvalue ratio $\rho=\lambda_{1} / \lambda_{2}$. If $\rho>0$, then $g$ is hyperbolic. If $\rho=1$ (formally, using generalized eigenvalues), then $g$ is the identity or it is parabolic. If $\rho=\exp (i \theta)$, then $g$ is a rotation by an angle of $\theta$. If $\rho=-1$, then $g$ is a reflection, and otherwise if $\rho<0$ then $g$ is a glide reflection.

As before, we let $\tau_{1}, \tau_{2}$, and $\tau_{3}$ be the braid generators, and we let

$$
\sigma_{3}=\tau_{2} \tau_{1} \quad \sigma_{4}=\tau_{3} \tau_{2} \tau_{1}
$$

In the basis
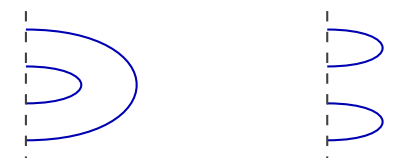

these elements have matrices

$$
\begin{aligned}
\tau_{1}=\tau_{3} & =\left(\begin{array}{cc}
-t^{1 / 4} & 0 \\
-t^{-1 / 4} & t^{-3 / 4}
\end{array}\right) \\
\tau_{2} & =\left(\begin{array}{cc}
t^{-3 / 4} & -t^{-1 / 4} \\
0 & -t^{1 / 4}
\end{array}\right)
\end{aligned}
$$




$$
\begin{aligned}
\sigma_{3} & =\left(\begin{array}{cc}
0 & -t^{-1} \\
1 & -t^{-1 / 2}
\end{array}\right) \\
\sigma_{4} & =\left(\begin{array}{cc}
0 & t^{-3 / 4} \\
t^{-3 / 4} & 0
\end{array}\right) .
\end{aligned}
$$

Suppose first that $t>0$. In this case, we use $\sigma_{3}$ and $\sigma_{4}$ as generators. The element $\sigma_{3}$ is a rotation by $2 \pi / 3$, while the element $\sigma_{4}$ is a reflection. Then $\sigma_{4}, \sigma_{3} \sigma_{4} \sigma_{3}^{-1}$, and $\sigma_{3}^{-1} \sigma_{4} \sigma_{3}$ are reflections through three lines that make a symmetric triangle $T$; however the triangle may have ideal or hyperideal vertices; see Figure 2(a) and Figure 2(b) for examples.

The structure of a vertex of the triangle can be determined from the element

$$
\left[\sigma_{3}, \sigma_{4}\right]=\tau_{1}^{-1} \tau_{2}=\left(\begin{array}{cc}
-t^{-1} & t^{-1 / 2} \\
-t^{-1 / 2} & 1-t
\end{array}\right)
$$

As written, this element has determinant 1 and trace $-t+1-t^{-1}$. It is equivalent to negate the trace. When $t+t^{-1}>3$, then the eigenvalues of $\gamma=\tau_{2}^{-1} \tau_{1}$ are real and positive, so that $\gamma$ is hyperbolic and the vertices of $T$ are hyperideal. In the marginal case that $t+t^{-1}=3, \gamma$ is parabolic. Finally when $t+t^{-1}<3, \gamma$ is elliptic. Using the formula

$$
t-1+t^{-1}=2 \cos \theta
$$

as $t$ goes to 1 from either side, $\theta$ goes monotonically to $\pi / 3$ from below.

It is easy to see that for all of the values of $t>0$ listed as discrete, the triangle $T$ tiles the hyperbolic plane $\mathbb{H}^{2}$ by reflections through its sides. $B_{4}$ acts on this tiling and the action is discrete. If we take the barycentric subdivision of each copy of $T$, the result is a tiling by a triangle $T^{\prime}$ with angles of $\pi / 2$ and $\pi / 3$, and either an ideal or a hyperideal vertex or an angle of $\theta / 2$. (See Figure 2(c).) The fundamental domain of the action of $B_{4}$ is either one or two copies of $T^{\prime}$.

The more subtle fact is that the action is not discrete when $\theta \neq 2 \pi / n$. This is known from the classification of 2-dimensional orbifolds.

Now let $t<0$. In this case we pass to the basis
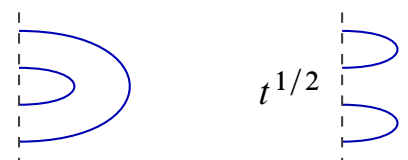
following the definition of $X(4 \cdot 1,0, t)_{\mathbb{R}}$. Now the matrices are

$$
\begin{aligned}
\tau_{1}=\tau_{3} & =\left(\begin{array}{cc}
-t^{1 / 4} & 0 \\
-t^{-3 / 4} & t^{-3 / 4}
\end{array}\right) \propto\left(\begin{array}{cc}
-t & 0 \\
1 & 1
\end{array}\right) \\
\tau_{2} & =\left(\begin{array}{cc}
t^{-3 / 4} & -t^{1 / 4} \\
0 & -t^{1 / 4}
\end{array}\right) \propto\left(\begin{array}{cc}
1 & -t \\
0 & t
\end{array}\right) \\
\sigma_{3} & =\left(\begin{array}{cc}
0 & -t^{-1 / 2} \\
t^{-1 / 2} & -t^{-1 / 2}
\end{array}\right) \propto\left(\begin{array}{cc}
0 & -1 \\
1 & -1
\end{array}\right) \\
\sigma_{4} & =\left(\begin{array}{cc}
0 & t^{-1 / 4} \\
t^{-5 / 4} & 0
\end{array}\right) \propto\left(\begin{array}{ll}
0 & t \\
1 & 0
\end{array}\right) .
\end{aligned}
$$

After rescaling to make the matrix real, $\operatorname{det} \sigma_{4}>0$ and $\sigma_{4}$ is a rotation by $\pi$ rather than a reflection. Meanwhile $\sigma_{3}$ is a rotation by $2 \pi / 3$ as before. The product $\tau_{1}=\sigma_{4} \sigma_{3}^{-1}$ has eigenvalue ratio $-t$; it is parabolic when $t=-1$ and is a translation otherwise. Thus the action of $T$ preserves the same tiling by $T^{\prime}$ as before, where here $T^{\prime}$ always has an ideal or hyperideal vertex. This time $B_{4}$ acts by the orientation-preserving subgroup of the symmetry group of the tiling.

Now consider case 3 . In this case $B_{4}$, and in particular $\sigma_{4}$, preserves the invariant, indefinite Hermitian form on $X(4 \cdot 1,0, t)$. Thus the action of $B_{4}$ is contained in a copy of $\operatorname{PSU}(1,1) \sim \operatorname{PSL}(2, \mathbb{R})$, and not just in its normalizer isomorphic to $\operatorname{PGL}(2, \mathbb{R})$. The analysis from the previous paragraph continues, except that the eigenvalue ratio $-t$ of $\tau_{1}$ now tells us that $\tau_{1}$ is a rotation by $\pi-|\theta|$. Again, when this is of the form $2 \pi / n$, the action of $B_{4}$ preserves a tiling of $T^{\prime}$, where $T^{\prime}$ is the finite triangle with angles of $\pi / 2, \pi / 3$, and $(\pi-|\theta|) / 2$. By the classification of 2-dimensional orbifolds, the action of $B_{4}$ is indiscrete for other choices of $\theta$.

Finally in case 4 , we once again use the element

$$
g=\left[\tau_{2}, \tau_{2} \tau_{3}^{3} \tau_{2} \tau_{1}^{-1}\right]
$$

with characteristic polynomial

$$
\begin{gathered}
\chi_{g}(x)=(x-1)\left(x^{2}+\left(t-1+t^{-1}\right)^{3} x+1\right) . \\
t+t^{-1}=1+2 \cos \frac{2 \pi}{7},
\end{gathered}
$$

When

the roots $\lambda$ other than 1 have the form $\exp (i \theta)$ with

$$
|\theta| \approx 165.812896^{\circ} .
$$

This time, $\lambda$ has degree at most 6 over $\mathbb{Q}$. If it did have order $s$, then $\phi(s) \leq 6$ so that $s \leq 18$. The approximation to $\theta$ shows that $\lambda$ is not a root of unity and $g$ is elliptic with infinite order. Thus by Lemma 3.11, the action of $B_{4}$ is indiscrete. 
Remark If $t=-1$ or $t=\exp ( \pm 2 \pi i / 3)$, then the action of $B_{4}$ on the space $W(4 \cdot 1,0, t)$ gives a natural extension of Theorem 1.4 since it is always 2-dimensional. When $t=-1$, the triangle $T^{\prime}$ has an ideal vertex and the projective action of $B_{4}$ is equivalent to $\operatorname{PSL}(2, \mathbb{Z})$. When $t=\exp ( \pm 2 \pi i / 3)$, then the action is equivalent to the orientation-preserving symmetries of the standard tiling of the Euclidean plane by equilateral triangles. Finally when $t=1$, the action is finite and equivalent to the symmetry group of a single triangle.

Proof of Corollary 1.5 It is well-known that the weighted Potts model of a planar graph $G$ can be realized within the Kauffman bracket by a skein replacement of every edge. Let $Z(G, n)$ be the Potts model with $n$ colors. We first let

$$
n=[2]^{2}=t+2+t^{-1} .
$$

Then we can make a Kauffman skein $S$ from $G$ so that

$$
Z(G, n)=(-[2])^{v}\langle S\rangle,
$$

where $G$ has $v$ vertices. The skein $S$ is obtained by replacing each vertex by doubling each incoming edge to two arcs, and stitching together these arcs into a planar matching:

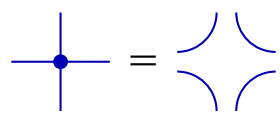

Then if an edge has Potts weight $y$, we can replace it as follows: e can replace an edge with Potts weight $y$ by

$$
\left.\stackrel{y}{\bullet} \cdot=\frac{1-y}{[2]}\right)(+\underset{\smile}{\smile} \text {. }
$$

In the case that $q>4$, we can take $t>1$ and directly apply case 4 of Corollary 1.2, because a crossing is proportional to a weighted edge with a real weight. This tells us that the action of the edge operators is real Zariski dense in $\operatorname{PSL}\left(V(n)_{\mathbb{R}}\right)$. At the same time, the action includes a Lie group of positive dimension, because the edge operator $A_{j, y}$ has a free parameter $y$. Thus the action includes all of $\operatorname{PSL}\left(V(n)_{\mathbb{R}}\right)$.

The case $q=4$ is more of a corollary of the proof of Theorem 1.1 and case 4 of Corollary 1.2. First, even though the corresponding value of the Jones polynomial is trivial, the Kauffman bracket still exists when $t=1$. Instead of using crossings, the proofs still hold using the replaced edge operator in Equation (3). This time, instead of an eigenvalue ratio of $t^{c+1}$, the eigenvalue ratio is unrestricted because $y$ is a free parameter. And the action includes a Lie group of positive dimension, so the action includes all of $\operatorname{PSL}\left(V(n)_{\mathbb{R}}\right)$. 


\section{References}

[1] D Aharonov, I Arad, E Eban, Z Landau, Polynomial quantum algorithms for additive approximations of the Potts model and other points of the Tutte plane arXiv: quant-ph/0702008

[2] A Borel, Linear algebraic groups, second edition, Graduate Texts in Math. 126, Springer, New York (1991) MR1102012

[3] E Breuillard, T Gelander, On dense free subgroups of Lie groups, J. Algebra 261 (2003) 448-467 MR1966638

[4] M H Freedman, MJ Larsen, $\mathbf{Z}$ Wang, The two-eigenvalue problem and density of Jones representation of braid groups, Comm. Math. Phys. 228 (2002) 177-199 MR1911253

[5] W Fulton, J Harris, Representation theory. A first course, Graduate Texts in Math. 129, Springer, New York (1991) MR1153249

[6] P Hall, The Eulerian functions of a group, Quart. J. Math. 7 (1936) 134-151

[7] L H Kauffman, S L Lins, Temperley-Lieb recoupling theory and invariants of 3manifolds, Annals of Math. Studies 134, Princeton Univ. Press (1994) MR1280463

[8] G Kuperberg, How hard is it to approximate the Jones polynomial? arXiv: 0908.0512

[9] D W Morris, Ratner's theorems on unipotent flows, Chicago Lectures in Math., Univ. of Chicago Press, Chicago, IL (2005) MR2158954

[10] S Roman, Field theory, second edition, Graduate Texts in Math. 158, Springer, New York (2006) MR2178351

Department of Mathematics, University of California

Davis CA 95616, USA

greg@math.ucdavis. edu

http://www . math.ucdavis.edu/ greg/

Proposed: Rob Kirby

Received: 16 September 2009

Seconded: Joan Birman, Mike Freedman

Accepted: 23 August 2010 